\title{
Spin-1/2 one- and two- particle systems in physical space without eigen-algebra or tensor product
}

Sokol Andoni ( $\square$ sond4p@gmail.com )

Technical University of Denmark https://orcid.org/0000-0002-1719-6789

\section{Research Article}

Keywords: spin 1/2, 3D subalgebra, spacetime algebra (STA), eigen-algebra, tensor product

Posted Date: January 20th, 2022

DOI: https://doi.org/10.21203/rs.3.rs-235048/v5

License: (9) This work is licensed under a Creative Commons Attribution 4.0 International License. Read Full License 


\section{Spin-1/2 one- and two- particle systems in physical space without eigen-algebra or tensor product}

Sokol Andoni*, Technical University of Denmark, Kgs. Lyngby 2800, Denmark

*Corresponding author, sond4p@gmail.com

Abstract. A novel representation of spin 1/2 combines in a single geometric object the standard Pauli spin operator and spin state. Under the spin-position decoupling approximation it consists of the sum of three orthogonal vectors comprising a gauge phase. In the one-spin case the representation: (1) is Hermitian; (2) endowed with handedness; (3) yields all standard results, including the total spin angular momentum $S=$ $\sqrt{3} \hbar / 2$; (4) relates basis spins by proper rotations, thus preserving handedness; (5) allows formalizing irreversibility in spin measurement. In the bipartite case: (1) entangled spins have precisely related gauge phases and opposite handedness; (2) maximally entangled spins relate by one of the four improper rotations in 3D: plane-reflections (triplets) and inversion (singlet); (3) the full spin expressions yield the standard total two-spin angular momentum; (4) all standard expected values for bipartite observations follow. Depending on whether spin operations act one - or two - sided, the formalism appears in two complementary forms, the 'spinor' or the 'vector' form, respectively. The proposed scheme provides a clear geometric picture of spin transformations and correlations in the 3D physical orientation space.

\section{Introduction}

Key developments in Quantum Mechanics (QM), such as the first phenomenological description of spin 1/2 by Pauli [1] and the first quantum relativistic description of the electron by Dirac [2], 're-invented' Clifford algebras (in the matrix representation), seemingly unaware of Grassmann's [3] and Clifford's [4] works more than half a century earlier. The promotion of vector-generated Clifford algebras in Physics and in particular the development of the spacetime algebra (STA) was undertaken by Hestenes $[5,6]$, with more scientists joining in during the last 2-3 decades [7-10]. Spin formalism is arguably the main application of STA in QM [7]. In the STA literature $[6,7]$ it has become customary to represent spin by a bivector or the spin vector normal to it, both of modulus $\hbar / 2$. Notably, such representations of spin with quantum number $s=1 / 2$ do not comprise a quantity corresponding to the standard Pauli's vector spin operator with modulus equal to the total quantum spin angular momentum $S_{\text {tot }}=\hbar \sqrt{s(s+1)}=\sqrt{3} \hbar / 2$. The model presented in the following reproduces all standard results for systems of one and two spin-half particles, naturally including $S_{\text {tot }}$. 
The rest of the report comprises three parts. I introduce basic concepts of geometric algebra in Part 2. The new definition of spin opens Part 3. Correlations for the one and two particle cases appear in it in the vector form, i.e. as two-sided operations. In Part 4 correlations for the same two cases will be demonstrated in the spinor form, i.e. as one-sided transformations. Orthogonality relations, e.g. between spin up and spin down hold in the spinor representation. The connection between the vector and the spinor forms is also discussed in Part 4 followed by Conclusions at the end.

\section{A Short Introduction to Geometric Algebra}

The geometric or Clifford product of two vectors $\mathbf{u}, \mathbf{v}$, let say in a 3D Euclidean space, combines Hamilton's scalar (symmetric) and Grassmann's wedge (antisymmetric) products; if not zero it is invertible and as it will become clear in definition (3) it is proportional to a rotor:

$\mathbf{u v}=\mathbf{u} \cdot \mathbf{v}+\mathbf{u} \wedge \mathbf{v}=\mathbf{v} \cdot \mathbf{u}-\mathbf{v} \wedge \mathbf{u} ;(\mathbf{u v})^{-1}=\mathbf{v u} / \mathbf{u}^{2} \mathbf{v}^{2}$. In terms of anti-commutator and commutator

brackets: $\{\mathbf{u}, \mathbf{v}\} \equiv \mathbf{u v}+\mathbf{v u}=2 \mathbf{u} \cdot \mathbf{v} ;[\mathbf{u}, \mathbf{v}] \equiv \mathbf{u v}-\mathbf{v u}=2 \mathbf{u} \wedge \mathbf{v}$.

The geometric product generalizes to any dimension and space signature, whereas the cross product is valid only in 3D. The two relate by $I(\mathbf{u} \times \mathbf{v})=\mathbf{u} \wedge \mathbf{v}$ (a bivector), with $I$ the pseudoscalar (see the basis (2)).

Recently I generalized STA - a Clifford algebra $\mathcal{C} \ell_{(1,3)}$ (signature $(+---)$ ) on a $16 \mathrm{D}$ real vector space, to spacetime - reflection (STR) - a $\mathcal{C} \ell_{(2,3)}$ algebra on a 32D real vector space X [11]. The equivalent real dimension of the space of Dirac matrices is also 32. Here I address spin in the non-relativistic regime under the spin-position decoupling approximation $[6,7,12]$. The 3D physical space in this case reduces to orientation space at a point (the origin) and the relevant symmetry operations are proper rotations and reflections. In STR it corresponds to the subspace $\boldsymbol{\Sigma}$ of $\mathrm{X}$ generated by the 'axial' Lorentz rotation vectors $\boldsymbol{\sigma}_{j} \equiv \mathrm{x}_{j 05}(j=1,2,3)[11]$ with a Clifford algebra $\mathcal{C} \ell_{3,0}$ isomorphic to that of Pauli matrices, therefore the notation $[9,11]\left(\boldsymbol{\sigma}_{j}\right.$ are parity-even, behaving as axial vectors in X). $\boldsymbol{\Sigma}$ differs from the even subspace of STA, the last coinciding in STR with the subspace $\mathbf{X}$ generated by the 'polar' boost vectors $\mathbf{x}_{j} \equiv \mathrm{x}_{j 0}$. However, $\boldsymbol{\Sigma}$ in STR and the even subspace in STA have same dimension and same algebra $\mathcal{C} \ell_{3,0}[7,11]$. A basis of $\boldsymbol{\Sigma}$ consists of one unit scalar, three orthonormal vectors, three bivectors and one trivector, or pseudoscalar, all unitless $\left(\delta_{j k}, \varepsilon_{j k l}\right.$ below are the Kronecker and the totally antisymmetric Levi-Civita symbols): 
$\left\{1(1), \boldsymbol{\sigma}_{j}(3), \boldsymbol{\sigma}_{j} \boldsymbol{\sigma}_{k} \equiv \boldsymbol{\sigma}_{j k}\left(=\delta_{j k}+\varepsilon_{j k l} I \boldsymbol{\sigma}_{l}\right)(3), \boldsymbol{\sigma}_{123} \equiv I(1)\right\} ; I \boldsymbol{\sigma}_{j}=\boldsymbol{\sigma}_{j} I ; j, k, l=1,2,3 ; \operatorname{dim} 8$

Remind that $\boldsymbol{\sigma}_{j}$ are vectors, not matrices. Geometrically, the bivectors $I \sigma_{l}$, e.g. $I \sigma_{2}=\sigma_{31}$, represent oriented surface elements, while the pseudoscalar $I=\sigma_{123}$ is an oriented volume element. A rotor $\mathrm{R}_{\vartheta}$ with axis along the unit vector $\mathbf{u}$ is a unitary transformation in $\boldsymbol{\Sigma}$ (length, angle and handedness preserving):

$\boldsymbol{\Sigma} \ni \mathrm{A} \rightarrow R_{\vartheta} \mathrm{A} R_{\vartheta}^{\dagger} \equiv \exp (-I \mathbf{u} \vartheta / 2) \mathrm{A} \exp (I \mathbf{u} \vartheta / 2) \in \boldsymbol{\Sigma} ; R_{\vartheta}=\cos \frac{\vartheta}{2}-I \mathbf{u} \sin \frac{\vartheta}{2}$

Rotors can alter vectors and bivectors in $\boldsymbol{\Sigma}$, not (pseudo)scalars. Inversion and plane-reflections are another type of transformations in $\boldsymbol{\Sigma}$, which also conserve lengths and angles, but invert handedness, e.g. of the triplet $\left(\sigma_{1}, \sigma_{2}, \sigma_{3}\right) \equiv\left\{\sigma_{j}\right\}$. The three frame reflections and the inversion can be depicted by $\left(\boldsymbol{\sigma}_{0}=1\right)$ :

$\boldsymbol{\Sigma} \ni \mathrm{A} \rightarrow I \boldsymbol{\sigma}_{\mu} \mathrm{AI} \boldsymbol{\sigma}_{\mu}=-\boldsymbol{\sigma}_{\mu} \mathrm{A} \boldsymbol{\sigma}_{\mu} \in \boldsymbol{\Sigma} ; \quad\left\{\boldsymbol{\sigma}_{j}\right\} \rightarrow I \boldsymbol{\sigma}_{\mu}\left\{\boldsymbol{\sigma}_{j}\right\} I \boldsymbol{\sigma}_{\mu}=(-1)^{\delta_{\mu 0}}\left\{(-1)^{\delta_{\mu j}} \boldsymbol{\sigma}_{j}\right\} \in \boldsymbol{\Sigma} ; \mu=0,1,2,3$

It is now appropriate to present the novel definition of spin $1 / 2$ in STR.

\section{Definition and vector (two-sided) transformations of spin $1 / 2$ for one and two particle systems.}

3.1. One-particle systems. Any spin $1 / 2$ is modelled here by the sum of three orthogonal vectors and a (nonobservable) phase $\varphi$. Specifically, spin up $\uparrow_{\sigma}\left(\right.$ down $\left.\downarrow_{\sigma}\right)$ along the $\sigma_{3}$ axis is the sum of three orthonormal vectors scaled by $\hbar / 2$ together with a two-sided rotor $R_{\varphi}$ in the plane $\sigma_{12}=I \sigma_{3}$ (see (3) and figure 1a):

$$
\begin{aligned}
& \mathrm{S}_{\uparrow \sigma}=\frac{\hbar}{2} R_{\varphi}\left(\boldsymbol{\sigma}_{3}+\boldsymbol{\sigma}_{1}+\boldsymbol{\sigma}_{2}\right) R_{\varphi}^{\dagger}=\frac{\hbar}{2}\left(\boldsymbol{\sigma}_{3}+R_{\varphi}\left(\boldsymbol{\sigma}_{1}+\boldsymbol{\sigma}_{2}\right) R_{\varphi}^{\dagger}\right) \equiv \frac{\hbar}{2}\left(\boldsymbol{\sigma}_{3}+\left(\boldsymbol{\sigma}_{1}+\boldsymbol{\sigma}_{2}\right)_{\varphi}\right) ; \\
& \mathrm{S}_{\downarrow \sigma}=\sigma_{2} \mathrm{~S}_{\uparrow \sigma} \boldsymbol{\sigma}_{2}=\frac{\hbar}{2}\left(-\boldsymbol{\sigma}_{3}+\left(-\boldsymbol{\sigma}_{1}+\boldsymbol{\sigma}_{2}\right)_{-\varphi}\right) ; R_{\varphi}=e^{-\frac{I \sigma_{3} \varphi}{2}}(\text { see }(3)) \\
& \mathrm{S}_{\uparrow} \mathrm{S}_{\uparrow}=\mathrm{S}_{\downarrow} \mathrm{S}_{\downarrow}=\mathrm{S}_{\sigma}^{2}=\frac{3 \hbar^{2}}{4} ; \mathrm{S}_{\sigma}^{\dagger}=\mathrm{S}_{\sigma} ; \mathrm{S}_{-\sigma} \neq-\mathrm{S}_{\sigma} ;\left\langle\mathrm{S}_{\sigma}\right\rangle \equiv \frac{\hbar}{2}\left( \pm \boldsymbol{\sigma}_{3}+\left\langle R_{ \pm \varphi}^{2}\right\rangle\left( \pm \boldsymbol{\sigma}_{1}+\sigma_{2}\right)\right)= \pm \frac{\hbar}{2} \sigma_{3}
\end{aligned}
$$

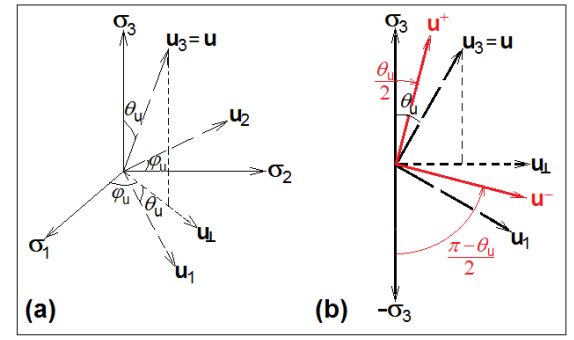

Figure 1. $3 D$ and $2 D$ rendering of $(a)$ the coordinate and (b) spinor transformation.
Spin handedness is assigned from the vector triplet (with signs as) in the expression for spin, so that $S_{\sigma}$ (i.e. $S_{\uparrow \sigma}$ or $S_{\downarrow \sigma}$ ) in (5) are both righthanded $(\boldsymbol{r})$, while inverted and plane-reflected $I \boldsymbol{\sigma}_{\mu} \mathrm{S}_{\sigma} I \boldsymbol{\sigma}_{\mu}$ (see (4)) are all left-handed $(\ell)$. The two subspaces $\{\ell\}$ and $\{r\}$ are disjoint under proper rotations, while reflections and inversion (improper rotations)

convert a spin from one space to the other, as already shown. Any left-handed $(\ell)$ spin-up (-down) is the inverse of a right-handed $(r)$ spin-down (-up), which shows the linear dependence in 3D between the two:

$S_{\sigma(\ell)}=I S_{-\sigma(r)} I=-S_{-\sigma(r)}$ 
We therefore choose + in linear relations between spins in order to keep track of handedness (see (20)). Reflection $\boldsymbol{\sigma}_{2} \mathrm{~S}_{\sigma} \boldsymbol{\sigma}_{2}$ onto $\boldsymbol{\sigma}_{2}$ is equivalent in $\boldsymbol{\Sigma}$ to a $\pi$-rotation of $S_{\sigma}$ around $\boldsymbol{\sigma}_{2}$. The phase $\varphi$ degree of freedom is unobservable, which I express here by the zero expected value $\langle\sin \varphi\rangle=\langle\cos \varphi\rangle=0$ (see last equation in (5)). In other words, measurement 'extracts' the phase-insensitive part of spin or combination of spins, see (8-8'), (13-14). Angled brackets denote either the expected value (a scalar) or the average (scalar, vector, etc.). I will also use the STA notation \langle\rangle$_{0}$ for the expected value, or the scalar part of an expression, if needed for clarity. The spin modulus $\sqrt{S_{\sigma}^{2}}=\sqrt{3} \hbar / 2$ from (5) directly relates to the QM total angular momentum of spin; it comprises equal contributions from the three vector components and exceeds by a factor of $\sqrt{3}$ the observed spin vector $(\hbar / 2) \boldsymbol{\sigma}_{3}$ with modulus $\sqrt{\left\langle S_{\sigma}\right\rangle^{2}}=\hbar / 2$. As clear from (5) $S_{\sigma}$ depicts either $S_{\uparrow \sigma}$ or $S_{\downarrow \sigma}$, while $S_{\sigma}$ and $S_{-\sigma}$ stand for opposite spins of same handedness. Focusing on $\{r\}$, any spin $S_{\mathrm{u}}$ can be expressed as a function of $S_{\sigma}$ and a combined rotation $R_{\mathrm{u}} \equiv R_{\theta_{\mathrm{u}}} R_{\varphi_{\mathrm{u}}}$ (see figures $1 \mathrm{a}, \mathrm{b}$ ):

$$
\begin{aligned}
& \left(S_{\mathrm{u}}\right)_{\varphi}=R_{\mathrm{u}}\left(S_{\sigma}\right)_{\varphi} R_{u}^{\dagger}=\frac{\hbar}{2} R_{\mathrm{u}}\left(\boldsymbol{\sigma}_{3}+\left(\boldsymbol{\sigma}_{1}+\boldsymbol{\sigma}_{2}\right)_{\varphi}\right) R_{\mathrm{u}}^{\dagger}=\frac{\hbar}{2} R_{\theta_{\mathrm{u}}}\left(\boldsymbol{\sigma}_{3}+\left(\mathbf{u}_{\perp}+\mathbf{u}_{2}\right)_{\varphi}\right) R_{\theta_{\mathrm{u}}}^{\dagger}= \\
& \frac{\hbar}{2}\left[\mathbf{u}+\left(\mathbf{u}_{1}+\mathbf{u}_{2}\right)_{\varphi}\right] ; R_{\theta_{\mathrm{u}}}=e^{\mathrm{B} \theta_{\mathrm{u}} / 2} ; \mathrm{B}=\mathbf{u}_{\perp} \boldsymbol{\sigma}_{3}=\mathbf{u}_{13}=\mathbf{u}^{-} \mathbf{u}^{+}=-I \mathbf{u}_{2}
\end{aligned}
$$

A measurement of $\left(S_{\sigma}\right)_{\varphi}$ by a Stern-Gerlach $(S G)$ magnet aligned along $\mathbf{u}=\mathbf{u}_{3}$, transforms spin with complete loss of phase correlation, as shown schematically by:

$$
\left(S_{\sigma}\right)_{\varphi} \stackrel{S G}{\rightarrow}\left(S_{\mathrm{u}}\right)_{\varphi^{\prime}} ; \quad\left\langle\sin \varphi^{\prime} \sin \varphi\right\rangle_{S G}=\left\langle\sin \varphi^{\prime}\right\rangle\langle\sin \varphi\rangle=0
$$

Then it is sufficient to restrict the transformation to only the phase-insensitive spin vector:

$\frac{\hbar}{2} \boldsymbol{\sigma}_{3} \rightarrow \frac{\hbar}{2} R_{\theta_{\mathrm{u}}} \boldsymbol{\sigma}_{3} R_{\theta_{\mathrm{u}}}^{\dagger}=\frac{\hbar}{2}\left(\mathbf{u}^{+} \cos \frac{\theta_{\mathrm{u}}}{2}+\mathbf{u}^{-} \sin \frac{\theta_{\mathrm{u}}}{2}\right)=\frac{\hbar}{2} \mathbf{u}$, where: $R_{\theta_{\mathrm{u}}} \boldsymbol{\sigma}_{3}=\mathbf{u}^{+} ; R_{\pi-\theta_{\mathrm{u}}}^{\dagger}\left(-\boldsymbol{\sigma}_{3}\right)=\mathbf{u}^{-}$

The halfway vectors $\mathbf{u}^{+}, \mathbf{u}^{-}$(see figure 1b) depict one form of reduced spinor representation and as shown by the last two equalities in (8') they arise by the action of one-sided rotors onto the spin vectors up and down, respectively. Of course, one can obtain both $\mathbf{u}^{+}$and $\mathbf{u}^{-}$by action of one-sided rotors on spin-up alone; even then, two terms are needed with distinct probability amplitudes for coincidence and anti-coincidence $\left( \pm \boldsymbol{\sigma}_{3}\right.$ are linearly dependent; $\mathbf{u}^{+}, \mathbf{u}^{-}$are not). For the sake of definiteness, we insist that a spin basis consist of two opposite spins; then they must have same handedness in order for the spinor representation illustrated in fig. $1 \mathrm{~b}$ to close into a proper rotation, as recounted in Part 4, eq. (20, 20'). Eqs. $\left(8,8^{\prime}\right)$ relate to spin measurement and probability amplitudes for coincidence/anticoincidence (more on spinor representation in Part 4). 
The expected value for the $S G$ transformation ( $8^{\prime}$ ) follows either from the correlation between start and end spins in (9), or from the projection of the start spin at the detector aligned along $\mathbf{u}$ in (9'):

$\frac{4}{\hbar^{2}}\left\langle S_{\sigma \mathrm{u}}\right\rangle_{0(S G)}=\frac{4}{\hbar^{2}}\left\langle\left\langle S_{\sigma}\right\rangle\left\langle S_{\mathrm{u}}\right\rangle\right\rangle_{0}=\left\langle\boldsymbol{\sigma}_{3} \mathbf{u}\right\rangle_{0}=\boldsymbol{\sigma}_{3} \cdot \mathbf{u}=\left(-\boldsymbol{\sigma}_{3}\right) \cdot(-\mathbf{u})=\cos \theta_{\mathrm{u}}=\cos ^{2} \frac{\theta_{\mathrm{u}}}{2}-\sin ^{2} \frac{\theta_{\mathrm{u}}}{2}$

$\frac{2}{\hbar}\left\langle S_{\sigma} \mathbf{u}\right\rangle_{0(S G)}=\left\langle\boldsymbol{\sigma}_{3} \mathbf{u}\right\rangle_{0}=\cos \theta_{\mathrm{u}}=\cos ^{2} \frac{\theta_{\mathrm{u}}}{2}-\sin ^{2} \frac{\theta_{\mathrm{u}}}{2}$

The probabilities for coincidence and anticoincidence outcomes are $\cos ^{2} \theta_{\mathrm{u}} / 2$ and $\sin ^{2} \theta_{\mathrm{u}} / 2$. (9') just tells that the detector alignment conditions the final spin axis. For later reference (see (17)), remark that (9) yields the expected value of one spin measurement by projecting out the wedge part of $\boldsymbol{\sigma}_{3} \mathbf{u}=\boldsymbol{\sigma}_{3} \cdot \mathbf{u}+\boldsymbol{\sigma}_{3} \wedge \mathbf{u}$. For bipartite measurements on pairs with correlated phases, the scalar parts still determine the expected value for 1-particle observations, but pair correlations comprise in addition a contribution from the wedge parts.

Now, if the relative probabilities and probability amplitudes show up in relations involving only spin vectors, like (8'), (9) and (19), why do we need the full spin (5)? Well, the full spin connects to the total quantum angular momentum of spin 1/2. In this sense, it is analogue to the standard Pauli spin vector. Also, opposite spin-vectors in 3D are linearly dependent, while the opposite full spins in (5) are not. As already told they relate by a unitary transformation, just as any two spins of same handedness also relate by unitary transformations, the two-sided rotors in (7), (20). In addition, the explicit gauge phase formalizes the entrance of irreversibility with measurement due to loss of phase correlation, thus resulting, as illustrated in (8), into expressions equivalent to the restricted transformations ( $\left.8^{\prime}\right),\left(23^{\prime}\right)$. While in the one-spin case one can pick one handedness or the other, in the two-spin case we need both with the full spin expression (5) becoming even more relevant as discussed in the next section. Phase and handedness are key concepts for understanding entanglements. In a sense, phase and handedness legitimate working in the 3D orientation space for the bipartite case, avoiding the tensor product. In order to render the formulation as clear as possible, I use an arrow $\stackrel{S G}{\rightarrow}$, or $\rightarrow$ only in relation to spin transformations by $S G$ measurements.

3.2. Two-particle systems. The above discussion for the one-particle case generalizes in the following way to two-particle systems, again preserving a clear geometric picture of spin. The total angular momentum of two spins is the vector sum of the single total angular momenta, i.e. (see (1) for the definition of scalar product):

$\mathrm{S}_{\text {tot }}=\mathrm{S}_{(1)}+\mathrm{S}_{(2)} ; \mathrm{S}_{\text {tot }}^{2}=\mathrm{S}_{(1)}^{2}+\mathrm{S}_{(2)}^{2}+\mathrm{S}_{(1)} \mathrm{S}_{(2)}+\mathrm{S}_{(2)} \mathrm{S}_{(1)}=\frac{3 \hbar^{2}}{2}+2 \mathrm{~S}_{(1)} \cdot \mathrm{S}_{(2)}$ 
$\mathrm{S}_{\text {tot }}$ is Hermitian and symmetric relative to the spin swap $\mathrm{S}_{(1)} \leftrightarrows \mathrm{S}_{(2)}$. Therefore, in the following discussion, which will revolve around equation (10), the shorthand $S_{(1)}, S_{(2)}$ will stand for $S_{(10 r 2)}, S_{(2 o r 1)}$, respectively. The maximally entangled (Bell) states [12] consist of spin pairs with the spins in each pair being specular or inverse images of each other. In terms of the basis spins $S_{\sigma}$ aligned to match $\mathrm{S}_{(1)}$ for a given pair they read:

$\Upsilon_{(\mu)}:\left\{S_{(1)} \equiv\left(S_{\sigma}\right)_{\varphi} ; S_{(2)}=\left(I \sigma_{\mu} S_{\sigma} \sigma_{\mu} I\right)_{\varphi} \mid\right.$ for $\mu=0,1,2,3$, resp. $\left.\Psi^{-}, \Phi^{+}, \Phi^{-}, \Psi^{+}\right\}$

We calculate now the scalars $\mathrm{S}_{\text {tot }}^{2}, \mathrm{~S}_{(1)} \cdot \mathrm{S}_{(2)}$ from (10) (below $\boldsymbol{\sigma}_{j} \mathrm{~S}_{\sigma} \boldsymbol{\sigma}_{j}=-\mathrm{S}_{\sigma}+2\left(\mathrm{~S}_{\sigma} \cdot \boldsymbol{\sigma}_{j}\right) \boldsymbol{\sigma}_{j}$, no sum on $j$ ):

$\mathrm{S}_{t o t(\mu)}=\mathrm{S}_{(1)}+\mathrm{S}_{(2)}=\left(\mathrm{S}_{\sigma}-\boldsymbol{\sigma}_{\mu} \mathrm{S}_{\sigma} \boldsymbol{\sigma}_{\mu}\right)_{\varphi}=\left\{\begin{array}{l}0 ;\left(\mathrm{S}_{t o t(0)}\right)^{2}=0 ; \\ 2\left[\mathrm{~S}_{\sigma}-\left(\mathrm{S}_{\sigma} \cdot \boldsymbol{\sigma}_{j}\right) \boldsymbol{\sigma}_{j}\right] ;\left(\mathrm{S}_{t o t(j)}\right)^{2}=2 \hbar^{2} ;\left(\Phi^{+}, \Phi^{-}, \Psi^{+}\right)\end{array}\right.$

$2\left(\mathrm{~S}_{(1)} \cdot \mathrm{S}_{(2)}\right)_{(\mu)}=-2\left(\mathrm{~S}_{\sigma}\right)_{\varphi} \cdot\left(\boldsymbol{\sigma}_{\mu} \mathrm{S}_{\sigma} \boldsymbol{\sigma}_{\mu}\right)_{\varphi}=\left\{\begin{array}{lr}-2 \mathrm{~S}^{2}=-3 \hbar^{2} / 2 ; & \left(\Psi^{-}\right) \\ 2\left[3 \hbar^{2} / 4-\hbar^{2} / 2\right]=\hbar^{2} / 2 ; & \left(\Phi^{+}, \Phi^{-}, \Psi^{+}\right)\end{array}\right.$

The paired spins relate by inversion in the singlet state $\Psi^{-}$and by plane-reflections in the three triplet states $\Psi^{+}, \Phi^{-}, \Phi^{+}$. The spin vector $\sigma_{3}$ of $S_{\sigma}$ is unknown and it varies randomly from one entangled pair to the next. Relations (11) mean that there exists a frame where spin takes the definition form (5) and that the entangled spins have identical phase. From the expression for total angular momentum $S^{2}=\hbar^{2} S(S+1)$, (11a, b) show that $S=1$ (or 0 ) - consistent with an observed combined spin of modulus $\sqrt{\langle\mathrm{S}\rangle^{2}}=\hbar$ (or 0 ) in the triplet (resp. singlet) states. Alternatively, looking at $\left\langle\mathrm{S}_{\mathrm{tot}}\right\rangle^{2}=\left(\left\langle\mathrm{S}_{(1)}\right\rangle+\left\langle\mathrm{S}_{(2)}\right\rangle\right)^{2}$ we find $\hbar^{2}$ (or 0) for $\Phi^{ \pm}$(resp. $\Psi^{ \pm}$), corresponding to the standard eigenvalues $\pm \hbar$ (resp. 0 ) along $\sigma_{3}$.

From the above discussion and following the convention $[12,13]$, we define explicitly $\Phi^{+}\left(\Phi^{-}\right)$to comprise two spin up (resp. down). One particular choice of mutually orthogonal states, as shown in the spinor form in Section 3.2 , is the following, with the handedness $(r, \ell)$ shown explicitly:

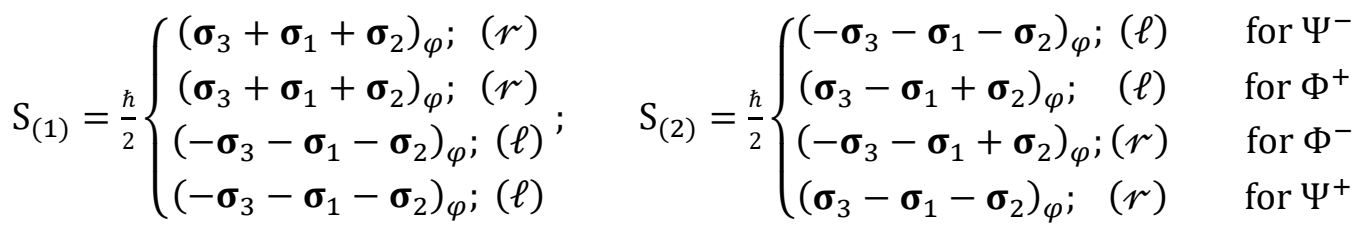

In (11') the spin sums for $\Phi^{+}, \Phi^{-}$lay on the respective cones defined by $\sigma_{3}+\left(\sigma_{2}\right)_{\varphi}$ and $-\sigma_{3}-\left(\sigma_{1}\right)_{\varphi}$ meeting at the origin and with a phase difference of $\pi / 2$; while the spin sum for $\Psi^{+}$lays in the plane $-\left(\sigma_{1}+\sigma_{2}\right)_{\varphi}$. Phase equality of entangled pairs is essential for the derivation of the results in (11-11b). 
'On-lab' transformations. Phase correlation between entangled pairs before observation is in the model also a necessary condition for calculating bipartite correlations under a common angled bracket. In order to reach at the standard form of results for the expected values, I change the perspective here relative to that chosen for the 'on paper' relations (11-11b); I select a fixed reference frame $\left\{\boldsymbol{\sigma}_{j}\right\}$ for the plane-reflections. This is physically motivated, as reflections in 'on lab' transformations have to apply relative to observed frame and spin axis - the last conditioned by the detector alignment. Schematically, observations with detectors aligned along $\mathbf{u}, \mathbf{v}$ can be depicted by (note, reflections apply to the observed spin $\mathrm{S}_{\mathrm{v}}$ !):

$\Upsilon_{(\mu)} \equiv\left\{\mathrm{S}_{(1)}, \mathrm{S}_{(2)}\right\}_{(\mu)} \stackrel{S G}{\rightarrow}\left\{\mathrm{S}_{\mathrm{u}}, I \boldsymbol{\sigma}_{\mu} \mathrm{S}_{\mathrm{v}} I \boldsymbol{\sigma}_{\mu}\right\}_{S G} ; \mu=0,1,2,3$ for the four Bell states.

The cross-product terms in (10) are the part of the squared total spin connecting to a measurement; therefore, the correlation expected values involve that part alone:

$$
\begin{gathered}
\left\langle\Upsilon_{(\mu)}\right\rangle_{S G} \equiv\left\langle\frac{2}{\hbar^{2}}\left(\mathrm{~S}_{(1)} \mathrm{S}_{(2)}+\mathrm{S}_{(2)} \mathrm{S}_{(1)}\right)_{(\mu)}\right\rangle_{S G}=-\frac{4}{\hbar^{2}}\left(R_{\mathrm{u}} \mathrm{S} R_{\mathrm{u}}^{\dagger}\right)_{S G} \cdot\left(\boldsymbol{\sigma}_{\mu} R_{\mathrm{v}} \mathrm{S} R_{\mathrm{v}}^{\dagger} \boldsymbol{\sigma}_{\mu}\right)_{S G}=-\mathbf{u} \cdot\left(\boldsymbol{\sigma}_{\mu} \mathbf{v} \boldsymbol{\sigma}_{\mu}\right)= \\
\left\{\begin{array}{c}
-(\mathbf{u} \cdot \mathbf{v})=-\cos \vartheta_{\mathrm{uv}}=\sin ^{2} \frac{\vartheta_{\mathrm{uv}}}{2}-\cos ^{2} \frac{\vartheta_{\mathrm{uv}}}{2} ; \\
\mathbf{u} \cdot \mathbf{v}-2\left(\mathbf{u} \cdot \boldsymbol{\sigma}_{j}\right)\left(\mathbf{v} \cdot \boldsymbol{\sigma}_{j}\right)=\sum_{k=1,2,3} u^{k} v^{k}-2 u^{j} v^{j}=\cos \vartheta_{\mathrm{uv}}-2 \cos \vartheta_{\mathrm{u} j} \cos \vartheta_{\mathrm{v} j} ; \mu=j=1,2,3
\end{array}\right.
\end{gathered}
$$

$\mathrm{S}$ stands for one of the unknown paired spins; $u^{k}, v^{k}$ are the scalar components of $\mathbf{u}$ and $\mathbf{v}$ along $\boldsymbol{\sigma}_{k}$, i.e. the expected values for triplet states depend on the reference frame. The bounds $-\hbar^{2} / 2 \leq\left(2 \mathrm{~S}_{(1)} \cdot \mathrm{S}_{(2)}\right)_{S G} \leq$ $\hbar^{2} / 2$ become explicit in (13) for all two-particle states. Also, the expected values do not change under swapped reflection $\mathrm{S}_{\mathrm{u}} \cdot\left(I \boldsymbol{\sigma}_{\mu} \mathrm{S}_{\mathrm{v}} I \boldsymbol{\sigma}_{\mu}\right)=\left(I \boldsymbol{\sigma}_{\mu} \mathrm{S}_{\mathrm{u}} I \boldsymbol{\sigma}_{\mu}\right) \cdot \mathrm{S}_{\mathrm{v}}$, as they should in respect of the spin swap symmetry $\mathrm{S}_{(1)} \leftrightarrows \mathrm{S}_{(2)}$. Both 'on paper' (11b) and 'on lab' (13) expectations satisfy the frame-independent 'closure' relation: $\sum_{\mu}\left(\mathrm{S}_{(1)} \cdot \mathrm{S}_{(2)}\right)_{(\mu)}=0$. The advantage of (13) is that the two terms render the spin swap symmetry explicit yielding directly the scalar product of the two spins; however, for calculation purposes, we could also have defined the expectation values by the scalar part of only one term:

$$
\left\langle\Upsilon_{(\mu)}\right\rangle_{S G} \equiv-\frac{4}{\hbar^{2}}\left\langle S_{\mathbf{u}} \boldsymbol{\sigma}_{\mu} S_{\mathbf{v}} \boldsymbol{\sigma}_{\mu}\right\rangle_{S G}=\left\{\begin{array}{c}
-(\mathbf{u} \cdot \mathbf{v}) \text { for }\left\langle\Psi^{-}\right\rangle(\mu=0) \\
\left(\mathbf{u} \cdot \mathbf{v}-2\left(\mathbf{u} \cdot \boldsymbol{\sigma}_{k}\right)\left(\mathbf{v} \cdot \boldsymbol{\sigma}_{k}\right)\right)(\mu=k=1,2,3)
\end{array} ; \quad \sum_{\mu}\left\langle\Upsilon_{(\mu)}\right\rangle=0\right.
$$

The form of correlation expectation value in (14) hints to a spinor representation of the four maximally entangled states, applied and discussed in Section 4.2 (see relations (24-25)). 
The derivation of $(13,14)$ shows that one first $S G$-transforms $\mathrm{S} \rightarrow \mathrm{S}_{\mathrm{u}}$ and $I S I \rightarrow-\mathrm{S}_{\mathrm{v}}$, rendering at the end the triplet states by $\pi$-rotations of $\mathrm{S}_{\mathrm{v}}\left(\right.$ or $\mathrm{S}_{\mathrm{u}}$ ) around each basis axis in $\left\{\boldsymbol{\sigma}_{j}\right\}$. This remark opens a 'shortcut' to the connection between one-spin and two-spin correlations in a bipartite measurement. We apply first the 1spin relation (9) to $S_{(1)}$ and $S_{(2)}$, showing in (17) the role of the separate wedge parts in 2-spin correlations:

$\frac{4}{\hbar^{2}}\left\langle\mathrm{~S}\left(R_{\mathrm{u}} \mathrm{S} R_{\mathrm{u}}^{\dagger}\right)_{S G(1)}\right\rangle=\left\langle\mathbf{s}_{3} \mathbf{u}\right\rangle ; \quad \frac{4}{\hbar^{2}}\left\langle\mathrm{~S}\left(I \boldsymbol{\sigma}_{\mu} R_{\mathrm{v}} \mathrm{S} R_{\mathrm{V}}^{\dagger} I \boldsymbol{\sigma}_{\mu}\right)_{S G(2)}\right\rangle=-\left\langle\mathbf{s}_{3}\left(\boldsymbol{\sigma}_{\mu} \mathbf{v} \boldsymbol{\sigma}_{\mu}\right)\right\rangle$

Now, for each separate one-spin observation (remind the geometric product, e.g. $\mathbf{s}_{3} \mathbf{u}=\mathbf{s}_{3} \cdot \mathbf{u}+\mathbf{s}_{3} \wedge \mathbf{u}$ ):

$(1):\left\langle\mathbf{s}_{3} \mathbf{u}\right\rangle_{0}=\left\langle\mathbf{s}_{3}\right\rangle \cdot \mathbf{u}=0$

$(2):-\left\langle\mathbf{s}_{3}\left(\boldsymbol{\sigma}_{\mu} \mathbf{v} \boldsymbol{\sigma}_{\mu}\right)\right\rangle_{0}=-\left\langle\mathbf{s}_{3}\right\rangle \cdot\left(\boldsymbol{\sigma}_{\mu} \mathbf{v} \boldsymbol{\sigma}_{\mu}\right)=0$

The zero expected values after $N \gg 1$ measurements follow from the randomness of the spin vector $\mathbf{s}_{3}$; for fixed $\mathbf{u}, \mathbf{v}, \boldsymbol{\sigma}_{k}$ there will be equal probability for spin up and spin down outcomes. Looking at two coupled spins, the phase correlation (11) before observation imposes on us to take under same angled bracket the contributions from the two spins $\left(\mathbf{s}_{3} \mathbf{u}\right.$ is not Hermitian) obtaining at the end a result equivalent to (14), i.e.:

$(1 \& 2):\left\langle\Upsilon_{(\mu)}\right\rangle_{S-G}=\left\langle\left(\mathbf{s}_{3} \mathbf{u}\right)^{\dagger} \mathbf{s}_{3} I \boldsymbol{\sigma}_{\mu} \mathbf{v} I \boldsymbol{\sigma}_{\mu}\right\rangle_{0}=-\left\langle\mathbf{u} \mathbf{s}_{3} \mathbf{s}_{3} \boldsymbol{\sigma}_{\mu} \mathbf{v} \boldsymbol{\sigma}_{\mu}\right\rangle_{0}=-\left\langle\mathbf{u} \boldsymbol{\sigma}_{\mu} \mathbf{v} \boldsymbol{\sigma}_{\mu}\right\rangle_{0}$

Truly (15-17) belong to one-sided (spinor-type) transformations, as we will see more of in Part 4.

As a final detail one can schematically characterize the entanglement states by the four (not unique) sign combinations below in front of the basis vectors; the first column of signs stands for $S_{(1)}\left(\right.$ see $\left.\left(11^{\prime}\right)\right)$ :

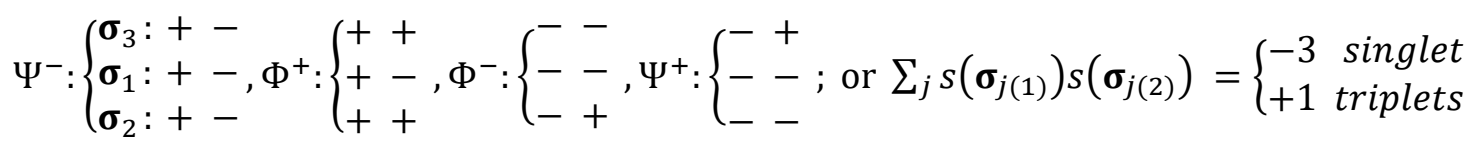

The last equality is a succinct expression for the 'on paper' scalar $S_{(1)} \cdot S_{(2)}$ in units of $\hbar^{2} / 4$, see $(11 b)$. What about the orthogonality of the maximally entangled states? Alike the one-particle case (remember $\mathbf{u}^{+}, \mathbf{u}^{-}$in fig. 1b), orthogonality is experienced in the spinor (one-sided rotor) representation, as described by equations (22-24) and the relative discussions in Section 4.2.

Relations (12-18) are invalid for non-entangled spins. In this case the gauge phases of the spins $\mathrm{S}_{(1)}$, $\mathrm{S}_{(2)}$ are not correlated before measurement and the two spins form a mixed state. For example, instead of the singlet state in (17), there will be a pair of antiparallel spins along the $\sigma_{3}$ axis, while the total spin will lie in the $\sigma_{12}$ plane and vary randomly between the limiting values of $\pm \hbar$. The expected value in this case will be the product of the separate expected values, instead of the expected value of the product in (17): 
$\left\langle S_{(1)} S_{(2)}\right\rangle_{0}=\frac{4}{\hbar^{2}}\left\langle\left\langle S_{\sigma}\right\rangle \mathbf{u}\right\rangle_{0}\left\langle\left\langle S_{-\sigma}\right\rangle \mathbf{v}\right\rangle_{0}=-\left(\boldsymbol{\sigma}_{3} \cdot \mathbf{u}\right)\left(\boldsymbol{\sigma}_{3} \cdot \mathbf{v}\right)=-u^{3} v^{3}=\cos \theta_{\mathrm{u}} \cos \theta_{\mathrm{v}}$

One future scope could be to adapt the present STR formalism to describe spin pairs between the maximally entangled states from relations (11-17) and the mixed states (19), as depending on the relative gauge phase.

\section{Spinor (one-sided rotor) representation of spin 1/2 one and two particle systems.}

In spinor form the transformation of a given spin appears as a sum (see (6)) of one-sided rotor operations of spin-up and spin-down with the standard probability amplitudes for coincidence and anti-coincidence.

4.1. One-particle systems. Instead of the two-sided rotor vector expression (7) we now express a given spin $S_{\mathrm{u}(\varphi)}$ as a sum (+ sign, see (6)) of the (one-sided rotor) spinor forms for the spin basis, as illustrated in Fig. $1 \mathrm{~b}$ (remind that the orientation of the angle $\theta_{\mathrm{u}}$ is as seen from $\mathbf{u}_{2}$ ):

$S_{\mathrm{u}\left(\varphi-\varphi_{\mathrm{u}}\right)}=R_{\theta_{\mathrm{u}}} S_{\sigma(\varphi)} \cos \frac{\theta_{u}}{2}+R_{\pi-\theta_{\mathrm{u}}}^{\dagger} S_{-\sigma(-\varphi)} \sin \frac{\theta_{u}}{2}=R_{\theta_{\mathrm{u}}} S_{\sigma(\varphi)} \cos \frac{\theta_{u}}{2}+$

$R_{(\pi-\theta)_{\mathrm{u}}}^{\dagger} R_{\pi_{\mathrm{u}}} S_{\sigma(\varphi)} R_{\pi_{\mathrm{u}}}^{\dagger} \sin \frac{\theta_{u}}{2}=R_{\theta_{\mathrm{u}}} S_{\sigma(\varphi)}\left(\cos \frac{\theta_{u}}{2}+I \mathbf{u}_{2} \sin \frac{\theta_{u}}{2}\right)=R_{\theta_{\mathrm{u}}} S_{\sigma(\varphi)} R_{\theta_{\mathrm{u}}}^{\dagger}$, or

$S_{\mathrm{u}\left(\varphi-\varphi_{\mathrm{u}}\right)}=\frac{\hbar}{2}\left[\left(\mathbf{u}^{+}+R_{\theta_{\mathrm{u}}}\left(\boldsymbol{\sigma}_{1}+\boldsymbol{\sigma}_{2}\right)_{\varphi}\right) \cos \frac{\theta_{u}}{2}+\left(\mathbf{u}^{-}-R_{\pi-\theta_{\mathbf{u}}}^{\dagger}\left(\boldsymbol{\sigma}_{1}-\boldsymbol{\sigma}_{2}\right)_{-\varphi}\right) \sin \frac{\theta_{u}}{2}\right]=$

$\frac{\hbar}{2}\left(\mathbf{u}^{+} \cos \frac{\theta_{u}}{2}+\mathbf{u}^{-} \sin \frac{\theta_{u}}{2}+R_{\theta_{\mathbf{u}}}\left(\left(\mathbf{u}_{\perp}+\mathbf{u}_{2}\right)_{\varphi-\varphi_{\mathbf{u}}} \cos \frac{\theta_{u}}{2}-I \mathbf{u}_{2}\left(\mathbf{u}_{\perp}-\mathbf{u}_{2}\right)_{-\varphi+\varphi_{\mathrm{u}}} \sin \frac{\theta_{u}}{2}\right)\right)=$

$\frac{\hbar}{2}\left(\mathbf{u}+R_{\theta_{\mathbf{u}}}\left(\mathbf{u}_{\perp}+\mathbf{u}_{2}\right)_{\varphi-\varphi_{\mathbf{u}}}\left(\cos \frac{\theta_{u}}{2}+I \mathbf{u}_{2} \sin \frac{\theta_{u}}{2}\right)\right)=\frac{\hbar}{2}\left(\mathbf{u}+R_{\theta_{\mathbf{u}}}\left(\mathbf{u}_{\perp}+\mathbf{u}_{2}\right)_{\varphi-\varphi_{\mathrm{u}}} R_{\theta_{\mathbf{u}}}^{\dagger}\right)=\frac{\hbar}{2}(\mathbf{u}+$

$\left.\left(\mathbf{u}_{1}+\mathbf{u}_{2}\right)_{\varphi-\varphi_{\mathbf{u}}}\right)$

By insisting that a basis consist of opposite spins then in order for $\left(20,20^{\prime}\right)$ to complete into a proper rotation as they do, the two spins must have same handedness. The plane for the phase angles in the subscripts follow the plane defined by the two vectors in brackets. The concise form at (20) is typical for STR/STA, where full geometric objects transform as a whole, with none or minimal need to work with the components as is the case for (20'). Keeping gauge phase implicit, the full spinor representations for spin up and spin down are:

$R_{\theta_{\mathrm{u}}} S_{\sigma}=\mathbf{u}^{+}+R_{\theta_{\mathrm{u}}}\left(\mathbf{u}_{\perp}+\mathbf{u}_{2}\right) \quad$ and $\quad I \mathbf{u}_{2} R_{\theta_{\mathrm{u}}} S_{-\sigma}=\mathbf{u}^{-}+R_{\theta_{\mathrm{u}}}\left(\mathbf{u}_{\perp}+\mathbf{u}_{2}\right) I \mathbf{u}_{2}$

Spinor representations are not spin according to definition (5). As already mentioned, the midway vectors $\mathbf{u}^{+}$and $\mathbf{u}^{-}$are reduced spinor representations of the two spins; they are manifestly orthonormal. Spinor representations are not unique. Another reduced spinor representation comprises the factors in front of the 
trigonometric functions of the rotor $R_{\theta_{\mathrm{u}}}$, a scalar 1 and a bivector $I \mathbf{u}_{2}$, respectively, which also appear in (21). These are even-grade elements of the 3D algebra and a standard choice in the STA literature [7]. In order for the reduced representation to be an orthonormal spinor basis one must have a zero scalar (grade 0 ) for $\left\langle 1\left(I \mathbf{u}_{2}\right)\right\rangle_{0}=\left\langle\mathbf{u}^{+} \mathbf{u}^{-}\right\rangle_{0}=0$, which is indeed the case. The orthogonality relation for the full spinor representation is (remember that $S_{\sigma}$ is Hermitian):

$\left\langle\left(R_{\theta_{\mathrm{u}}} S_{\sigma} I \mathbf{u}_{2}\right)^{\dagger}\left(R_{\theta_{\mathrm{u}}} S_{\sigma}\right)\right\rangle_{0}=-\left\langle\frac{3 \hbar^{2}}{4} I \mathbf{u}_{2}\right\rangle_{0}=0$, which is clearly satisfied

The orthogonality condition for the reduced representation is a normalized version of (22).

Measurement. The action of a $S G$ magnet aligned along $\boldsymbol{\sigma}_{3}$ on a spin with original spin vector $\hbar \mathbf{u} / 2,(\mathbf{u}=$ $\mathbf{u}_{3}$ ) corresponds to an irreversible transformation relative to the gauge phase and instead of (20') we get:

$S_{\mathrm{u}(\varphi)} \stackrel{S G}{\rightarrow} R_{\theta_{\mathrm{u}}}^{\dagger} S_{\mathrm{u}\left(\varphi^{\prime}\right)}=S_{\sigma\left(\varphi^{\prime}\right)} \cos \frac{\theta_{u}}{2}+I \mathbf{u}_{2} S_{-\sigma(-\varphi \prime)} \sin \frac{\theta_{u}}{2} ; \quad\left\langle\varphi \varphi^{\prime}\right\rangle=\langle\varphi\rangle\left\langle\varphi^{\prime}\right\rangle$

In the 'on lab' case it is straightforward to apply the spinor transformation to the spin vector alone, see (20'):

$\frac{\hbar}{2} \mathbf{u} \stackrel{S G}{\rightarrow} \frac{\hbar}{2} R_{\theta_{\mathbf{u}}}^{\dagger} \mathbf{u}=\frac{\hbar}{2} \boldsymbol{\sigma}_{3}\left(\cos \frac{\theta_{u}}{2}+I \mathbf{u}_{2} \sin \frac{\theta_{u}}{2}\right)=\frac{\hbar}{2} R_{\theta_{\mathbf{u}}}^{\dagger}\left(\mathbf{u}^{+} \cos \frac{\theta_{u}}{2}+\mathbf{u}^{-} \sin \frac{\theta_{u}}{2}\right)$

It is easy to revert (23') for a $S G$ aligned along $\mathbf{u}$ and incoming spin vector $\hbar \boldsymbol{\sigma}_{3} / 2$. The $S G$ transformation of spin vectors (23') is reversible, unlike the corresponding transformation of the phase-sensitive full spins (23).

4.2. Two-particle systems. By writing the non-normalized expression in (14) for $\mathbf{u}=\mathbf{v}=\boldsymbol{\sigma}_{3}$ as:

$\left\langle S_{\sigma} I \boldsymbol{\sigma}_{\mu} S_{\sigma} I \boldsymbol{\sigma}_{\mu}\right\rangle_{0}=\left\langle\left(-I \boldsymbol{\sigma}_{\mu} S_{\sigma}\right)^{\dagger}\left(\mathrm{S}_{\sigma} I \boldsymbol{\sigma}_{\mu}\right)\right\rangle_{0}$ (notice that $\mathrm{S}_{\sigma} I \boldsymbol{\sigma}_{\mu}$ is not Hermitian)

one realizes that a non-normalized spinor form for the entangled pairs consists of the conjugate pair:

$\Upsilon_{(\mu)(1 o r 2)} \equiv-I \sigma_{\mu} S_{\sigma} ; \Upsilon_{(\mu)(2 o r 1)} \equiv S_{\sigma} I \sigma_{\mu}=I \sigma_{\mu} \sigma_{\mu} S_{\sigma} \sigma_{\mu} \equiv I \sigma_{\mu} S_{\sigma}^{\prime}$

The spinor representations (25) for the four states comprise only terms that are either even (bivectors) for the singlet state $(\mu=0)$ or odd (vectors and pseudoscalar) for the triplet states $(\mu=j)$, therefore:

$\left\langle\Upsilon_{(0)}^{\dagger} \Upsilon_{(j)}\right\rangle_{0}=\left\langle\Upsilon_{(j)}^{\dagger} \Upsilon_{(0)}\right\rangle_{0}=0 \quad$ for both 'on paper' and 'on lab'

In words, the singlet state is orthogonal to the triplet states. As a curiosity, by taking the spins at the left (resp. right) in (11') as $S_{\sigma}\left(S_{\sigma}^{\prime}\right.$ from (25)), one can prove mutual orthogonality for all pairs of states $(\mu \neq v)$ : 
$\left\langle Y_{(\mu)}^{\dagger} \Upsilon_{(v)}\right\rangle_{0}=\left\langle\mathrm{S}_{\sigma} I \boldsymbol{\sigma}_{\mu}\left(I \boldsymbol{\sigma}_{v} \mathrm{~S}_{\sigma}^{\prime}\right)\right\rangle_{0}=-\left\langle\mathrm{S}_{\sigma} \boldsymbol{\sigma}_{\mu \nu} \mathrm{S}_{\sigma}^{\prime}\right\rangle_{0}=\left\langle C \mathrm{C}_{\sigma} \boldsymbol{\sigma}_{l} \mathrm{~S}_{\sigma}^{\prime}\right\rangle_{0}= \pm \hbar^{2} / 4\left\langle\mathrm{C}\left(\boldsymbol{\sigma}_{3}+\boldsymbol{\sigma}_{1}+\boldsymbol{\sigma}_{2}\right) \boldsymbol{\sigma}_{l}\left(\boldsymbol{\sigma}_{3}+\boldsymbol{\sigma}_{1}+\right.\right.$

$\left.\left.\boldsymbol{\sigma}_{2}\right)\right\rangle_{0}=0 ; \mathrm{C}=1$ for $\mu \nu=0 ; \mathrm{C}=I \varepsilon_{j k l}$ for $\mu \nu=j k ; j, k, l=1,2,3$

Finally, the orthogonality relations among the entangled states 'on lab' are straightforward:

$\left\langle Y_{(\mu)}^{\dagger} \Upsilon_{(v)}\right\rangle_{0(S-G)}=\left\langle\boldsymbol{\sigma}_{3} I \boldsymbol{\sigma}_{\mu}\left(I \boldsymbol{\sigma}_{v} \boldsymbol{\sigma}_{v} \boldsymbol{\sigma}_{3} \boldsymbol{\sigma}_{v}\right)\right\rangle_{0}= \pm\left\langle\boldsymbol{\sigma}_{\mu} \boldsymbol{\sigma}_{v}\right\rangle_{0}=0$ for $\mu \neq v ; \mu, v=0,1,2,3$

From $(27,28)$ the 'on paper' and 'on lab' spinor representations for the maximally entangled states are mutually orthogonal. The corresponding reduced spinor representations $\pm I \sigma_{\mu}$ are manifestly orthonormal for the four entanglement states $\Upsilon^{\mu}, \mu=0,1,2,3$, which is equivalent to equations (28). We do not need calculate the expected values for entangled pairs in spinor representation as by construction they are equal to $(13,14)$. A swift comparison of the two-particle spinor representation (25) with the vector representation (11) reveals that inversion and reflection operations apply to one of the spins in (11); the same operations split between the two spinor representations in (25). This makes states in (25) apparently more symmetric but also more abstract then the states in (11). However, as pointed out in relation to expression (10) there is full symmetry under the exchange $S_{(1)} \leftrightarrows S_{(2)}$. In addition, the cross-product term in the form (14) is the same for both representations and as a correlation expectation value it takes account of both entangled spins under the same angled brackets. This would represent a nonlocal operation, as the two measurements actually take place at different locations. The common angled brackets refer to the statistical dependence between the two measurements, directly connected to the correlation between gauge phases at the (local) event of creation of entangled pairs. Anyway, it is relevant to remind here that we are working in the spin-position decoupling approximation and in this framework the operation of common angled brackets is local. Notice that the bipartite states respect Pauli's exclusion principle, as they had to 'at the pairs' birth'.

\section{Conclusions}

All the above results were obtained by direct use and transformation (rotation, reflection) of spin in the 3D orientation space, without invoking concepts like eigen algebra and tensor product, which seem so fundamental in the standard formulation of QM. The 3D settings also provide a clear geometric meaning for the 
four Bell states: one singlet state of spin pairs related by inversion and three triplet states, each comprising spin pairs related by reflection onto one of the three coordinate planes of a chosen reference frame.

The STA spin model in (5) - sum of three orthogonal vectors with a gauge phase, displays many attractive features in the spin-position decoupling approximation. For one and two particle systems the same 3D geometric object (5) shows the correct representation of spin 1/2 relative to both 'on paper' and 'on lab' expectations. In a STA/STR context (5) merges the roles played by the Pauli spin matrix-vector and spin states in the standard formalism. The explicit gauge phase in (5) allows formalizing the irreversibility related to measurement ('on lab' transformation) as due to loss of phase correlation. Two entangled spins relate by improper rotations and show precise correlations of gauge phases, which implies that the bipartite expected values have to be calculated under the same angled brackets. A clear geometric picture can be maintained throughout the process of 'on paper' and 'on lab' transformations, not the least due to STR/STA's distinguished ability to work with whole geometric objects instead of components.

\section{References}

[1] Pauli, W.: Zur Quantenmechanik des magnetischen Elektrons. Zeitschrift für Physik, 43 (9-10), 601-623 (1927).

[2] Dirac, P.A.M.: The quantum theory of the electron. Proc. Roy. Soc. Lon. A, 117:610 (1928).

[3] Grassmann, H.: Die Ausdehnungslehre. Enslin, Berlin (1862).

[4] Clifford, W.K.: Applications of Grassmann's extensive algebra. Am. J. Math., 1:350 (1878).

[5] Hestenes, D.: Space-Time Algebra. Gordon and Breach, New York (1966).

[6] Hestenes, D.: Oersted Medal Lecture 2002: Reforming the Mathematical Language of Physics. Am. J. Phys. 71, 104121 (2003).

[7] Doran, C., Lasenby, A.: Geometric Algebra for Physicists, Cambridge University Press, Cambridge (2007).

[8] Doran, C.J.L., Lasenby, A.N. and Gull, S.F.: States and operators in the spacetime algebra. Found. Phys., 23(9):1239 (1993).

[9] Doran, C., Lasenby, A., Gull, S., Somaroo, S., Challinor, A.: Spacetime Algebra and Electron Physics. Adv. Imag. \& Elect. Phys. 95, 271-365 (1996). 
[10] Lasenby, A.: Geometric Algebra as a Unifying Language for Physics and Engineering and Its Use in the Study of Gravity. Adv. Appl. Clifford Algebras 27, 733-759 (2017).

[11] Andoni, S.: Dirac Equation Redux by Direct Quantization of the 4-Momentum Vector, DOI: 10.21203/rs.3.rs313921/v4, submitted to this Special Issue.

[12] Merzbacher, E.: Quantum Mechanics, J. Wiley \& Sons, New York, Chichester, Weineheim, Brisbane, Singapore, Toronto (1998).

[13] Bohm, D.: Quantum Theory (17.9), Prentice-Hall, Inc., Englewood Cliffs, New Jersey (1951). 